\title{
Bayesian denoising framework of phonocardiogram based on a new dynamical model
}

\author{
A. Almasi ${ }^{\text {a }}$, M. Bagher Shamsollahi ${ }^{\mathrm{a}}$, L. Senhadji ${ }^{\mathrm{b}, *, \mathrm{c}}$ \\ a Biomedical Signal and Image Processing Laboratory (BiSIPL), School of Electrical Engineering, Sharif University of Technology, Tehran, Iran \\ ${ }^{\mathrm{b}}$ Université de Rennes 1, LTSI, 35000 Rennes, France \\ ' Inserm, U 1099, 35000 Rennes, France
}

Received 1st April 2012; received in revised form 10 January 2013; accepted 29 January 2013

Available online 13 May 2013

\begin{abstract}
In this paper, we introduce a model-based Bayesian denoising framework for phonocardiogram (PCG) signals. The denoising framework is founded on a new dynamical model for PCG, which is capable of generating realistic synthetic PCG signals. The introduced dynamical model is based on PCG morphology and is inspired by electrocardiogram (ECG) dynamical model proposed by McSharry et al. and can represent various morphologies of normal PCG signals. The extended Kalman smoother (EKS) is the Bayesian filter that is used in this study. In order to facilitate the adaptation of the denoising framework to each input PCG signal, the parameters are selected automatically from the input signal itself. This approach is evaluated on several PCGs recorded on healthy subjects, while artificial white Gaussian noise is added to each signal, and the SNR and morphology of the outputs of the proposed denoising approach are compared with the outputs of the wavelet denoising (WD) method. The results of the EKS demonstrate better performance than WD over a wide range of PCG SNRs. The new PCG dynamical model can also be employed to develop other model-based processing frameworks such as heart sound segmentation and compression.
\end{abstract}

(C) 2013 Elsevier Masson SAS. All rights reserved.

\section{Introduction}

The phonocardiogram (PCG) signal contains valuable information about the human heart as it provides a quantitative and graphical representation of the acoustic waves produced by the mechanical activity of the heart. Generally, heart sounds and murmurs are two kinds of the acoustic vibrations of the heart. Heart sounds consist of two audible consecutive components known as $\mathrm{S} 1$ and $\mathrm{S} 2$, and occasionally two more components S3 and S4. These sounds are due to opening and closure of heart valves and also to vibrating cardiovascular structures. The murmurs are noise-like signals, a consequence of turbulent blood flow, and are usually signs of pathologic changes in the heart. Cardiac auscultation is a basic clinical tool to detect heart sounds and has a long history in cardiology. The electrocardiogram (ECG) signal, on the other hand, reveals the electrical activity of the heart and has useful information that is used to assess the condition of the heart. The ECG is not normally used unless

\footnotetext{
* Corresponding author.

E-mail address: lotfi.senhadji@univ-rennes1.fr (L. Senhadji).
}

a problem has been previously detected by auscultation, since setting up an ECG recording is a time-consuming process and is thus not used as a standard test; while PCG is easily obtained by placing the stethoscope against the skin. But PCG signals are usually corrupted by some sources of noise, such as breath sounds, contact of the recording microphone with the skin, fetal heart sounds if the subject is pregnant, and ambient noise. In fact, a much more useful diagnostic tool becomes available as soon as the unwanted noise is removed from the PCG.

In the past decade, great advances in ECG signal processing have been achieved that are originated from a novel dynamical model for ECG signal [1]. The ECG dynamical model introduced by McSharry et al. [1] was primarily intended for assessing biomedical signal processing techniques used to compute clinically relevant statistics from the ECG. Afterwards, Sameni et al. [2], and Sayadi et al. [3,4], by use of this model and nonlinear Bayesian filtering framework, created a model-based processing framework for ECG. Effectiveness of this approach motivates us to create a similar framework for PCG processing.

In a recent work [5], the authors suggested a morphologybased dynamical model capable of generating synthetic PCG, which was inspired by McSharry's dynamical model for ECG. 
In the current study, the synthetic PCG model is further discussed and then this approach is explored to establish a model-based processing framework for PCG denoising.

The paper is organized as follows. In Section 2 the ECG dynamical model proposed by McSharry et al. [1] and the modelbased ECG processing are briefly reviewed. This section also provides the necessary background of the Bayesian filtering that is used in the paper. PCG morphology and proposed dynamical model are presented in Section 3. The details of the proposed model-based denoising framework are presented in Section 4. Section 5 provides the simulation results of synthetic PCG generator and model-based denoising framework. A description of dataset and PCG wavelet denoising approach used as a benchmark for the proposed method are also presented in Section 5 , and finally concluding remarks and some perspectives are reported in Section 6.

\section{Review of electrocardiogram dynamical model and model-based electrocardiogram processing framework}

\subsection{Electrocardiogram dynamical model}

In 2003, McSharry et al. [1] proposed a dynamical model for generating realistic synthetic ECG signal using a set of state equations that generates a three-dimensional (3-D) trajectory in a 3-D state space with coordinates $(x, y, z)$. The model consists of a circular limit cycle of unit radius in the $(x, y)$ plane around which the trajectory is pushed up and down as it approaches the P, Q, R, S and T points in the ECG. Quasi-periodicity of the ECG is reflected by the movement of the trajectory around the attracting limit cycle. The dynamical equations of motion are given by a set of three ordinary differential equations in Cartesian coordinates:

$$
\begin{aligned}
& \dot{x}=\gamma x-\omega y \\
& \dot{y}=\gamma y+\omega x \\
& \dot{z}=-\sum_{i \in\{P, Q, R, S, T\}} a_{i} \Delta \theta_{i} \exp \left(-\frac{\Delta \theta_{i}^{2}}{2 b_{i}^{2}}\right)-\left(z-z_{0}\right)
\end{aligned}
$$

where $\gamma=\sqrt{x^{2}+y^{2}}, \Delta \theta i=\left(\theta-\theta_{i}\right) \bmod (2 \pi), \theta=\operatorname{atan} 2(y, x)$ is the four quadrant arctangent of the elements of $x$ and $y$, ranging over $[-\pi, \pi]$, and $\omega$ is the angular velocity of the trajectory as it moves around the limit cycle, and is related to the beat-to-beat heart rate as $\omega(t)=\frac{2 \pi}{R(t)}$ where $R(t)$ represents the time series generated by the RR-intervals. $x$ and $y$ in (1) are in fact the $(x$, $y$ ) components of Cartesian coordinates system and the first two equations in (1) represent the limit cycle of unit radius which moves around the origin with angular velocity $\omega$. The baseline wander of the ECG is modeled with the parameter $z_{0}$ that is assumed to be a relatively low amplitude sinusoidal component coupled with the respiratory frequency $f_{2}$ using

$z_{0}=A \sin \left(2 \pi f_{2} t\right)$

where $\mathrm{A}=0.15 \mathrm{~m} \mathrm{~V}$.

By neglecting the baseline wander term $\left(z-z_{0}\right)$ in (1), and integrating $\dot{z}$ equation, it can be seen that each component of the
Table 1

Typical parameters of the synthetic electrocardiogram (ECG) model [1].

\begin{tabular}{llllll}
\hline Index (i) & $\mathrm{P}$ & $\mathrm{Q}$ & $\mathrm{R}$ & $\mathrm{S}$ & $\mathrm{T}$ \\
\hline Time (s) & -0.2 & -0.05 & 0 & 0.05 & 0.3 \\
$\theta_{i}$ (radians) & $-\pi / 3$ & $-\pi / 12$ & 0 & $\pi / 12$ & $\pi / 2$ \\
$a_{i}$ & 1.2 & -5 & 30 & -7.5 & 0.75 \\
$b_{i}$ & 0.25 & 0.1 & 0.1 & 0.1 & 0.4 \\
\hline
\end{tabular}

ECG waveform is modeled with a Gaussian kernel which has three parameters $\theta_{i}, a_{i}, b_{i}$.

Values of the model parameters $\left(\theta_{i}, a_{i}, b_{i}\right)$ for each ECG beat can be obtained by curve fitting. Typical values of these parameters for the $\mathrm{P}, \mathrm{Q}, \mathrm{R}, \mathrm{S}$ and $\mathrm{T}$ points taken from [1] are shown in Table 1 . The times and angles are relative to the position of the R-peak since it is always assumed to have zero phase and the ECG contents lying between two consecutive R-peaks are assumed to have a phase between, $-\pi$ and $\pi$. The dynamic state equations proposed by (1) can also be written in polar coordinates as follows [2]:

$\dot{r}=r(1-r)$

$\dot{\theta}=\omega$

$\dot{z}=-\sum_{i \in\{P, Q, R, S, T\}} \frac{\alpha_{i} \omega}{b_{i}^{2}} \Delta \theta_{i} \exp \left(-\frac{\Delta \theta_{i}^{2}}{2 b_{i}^{2}}\right)-\left(z-z_{0}\right)$

In the above equation, the $a_{i}$ terms in (1) are replaced with:

$a_{i}=\frac{\alpha_{i} \omega}{b_{i}^{2}} \quad, \quad i \in\{P, Q, R, S, T\}$

where $\alpha_{i}$ are the peak amplitude of Gaussian kernels used for modeling each component of the ECG.

The new set of equations has some benefits compared to (1). The first equation in (3) shows the circular behavior of the generated trajectory by the model, which for any initial value of $r \geq 1$ reaches to a steady state of $r=1$ representing the limit cycle. The phase parameter $\theta$ has an explicit representation and indicates the angular location of the trajectory generated by the dynamical model (Table 1). On the other hand, the second and third equations in (3) are independent from $r$, making the first equation redundant. Therefore, the first equation may be excluded, as it has no effect on the synthetic ECG.

\subsection{Model-based electrocardiogram processing}

For the first time, Sameni et al. [2] used the ECG dynamical model to denoise ECG signal. They discretized the dynamical model of (3), with the assumption of a small sampling period of $\delta$ as:

$\theta_{k+1}=\left(\theta_{k}+\omega \delta\right) \bmod (2 \pi)$

$z_{k+1}=-\sum_{i} \delta \frac{\alpha_{i} \omega}{b_{i}^{2}} \Delta \theta_{i} \exp \left(-\frac{\Delta \theta_{i}^{2}}{2 b_{i}^{2}}\right)+z_{k}+\eta$ 
where $\Delta \theta_{i}=\left(\theta_{k}-\theta_{i}\right) \bmod (2 \pi), \eta$ is a random additive noise that models the inaccuracies of the dynamical model (including the baseline wander), and the summation over $i$ is taken over the number of Gaussian functions used for modeling the shape of ECG signal. Then, they combined the discrete version of the model with Kalman Filter concept and devised a framework for ECG denoising. In this framework, the phase signal $\theta$ and the clean ECG signal $z$ are considered as state variables, and the parameters of Gaussian functions as process noises. The noisy ECG and the approximated phase signal calculated from the RR-series are considered as observations.

Sayadi et al. [3] used this model-based processing framework for ECG compression and denoising. For this purpose, a first order auto-regressive dynamic was given to each parameter of the Gaussian functions and then they were included as state variables (but with no explicit observation). This technique facilitates the estimation of these parameters by the filter. Thus, the segmentation of ECG is possible by means of this framework [4].

\subsection{Review of the extended Kalman filter and Kalman smoother}

\subsubsection{Extended Kalman filter}

Filtering and estimation are two of the most pervasive tools of engineering. Estimation of the hidden states of a system with an underlying dynamic model is a typical problem in estimation theory. The Kalman filter (KF) is one of the most widely used methods for such purposes when the system dynamics and observation equations are linear. However, most systems are in practice nonlinear and so suitable extensions to the KF have been sought. The extended Kalman filter (EKF) is the most common approach for nonlinear systems, which simply linearizes all nonlinear equations of the model.

The dynamic model of a nonlinear system, with state vector $\mathbf{X}_{k}$ and observation vector $\mathbf{Y}_{k}$ at time instant $k$, can be represented as follows:

$$
\begin{aligned}
& \boldsymbol{X}_{k}=\boldsymbol{F}_{k}\left(\boldsymbol{X}_{k-1}, \boldsymbol{W}_{k}\right) \\
& \boldsymbol{Y}_{k}=\boldsymbol{G}_{k}\left(\boldsymbol{X}_{k}, \boldsymbol{V}_{k}\right)
\end{aligned}
$$

where $\mathbf{W}_{k}$ and $\mathbf{V}_{k}$ are the process and observation noise vectors respectively, with associated covariance matrices $Q_{k}=$ $E\left\{\mathbf{W}_{k} \mathbf{W}_{k}^{T}\right\}-\overline{\mathbf{W}}_{k} \overline{\mathbf{W}}_{k}^{T}$ and $R_{k}=E\left\{\mathbf{V}_{k} \mathbf{V}_{k}^{T}\right\}-\overline{\mathbf{V}}_{k} \overline{\mathbf{V}}_{k}^{T}$, where $\overline{\mathbf{W}}_{k}=E\left\{\mathbf{W}_{k}\right\}$ and $\overline{\boldsymbol{V}}_{k}=E\left\{\boldsymbol{V}_{k}\right\}$. The $F_{k}($.) represents the dynamic model of the state vector and $G_{k}($.$) characterizes the$ relationship between state variables and the observations. To use the KF relations for this system, a linearized version of the all nonlinear system equations must be derived. The linearization of (6) leads to:

$$
\begin{aligned}
& \mathbf{X}_{k} \approx F_{k}\left(\widehat{\mathbf{X}}_{k-1}, \overline{\mathbf{W}}_{k}\right)+A_{k}\left(\widehat{\mathbf{X}}_{\mathbf{k}-1}-\widehat{\mathbf{X}}_{k-1}\right)+B_{k}\left(\mathbf{W}_{k}-\overline{\mathbf{W}}_{k}\right) \\
& \mathbf{Y}_{k} \approx G_{k}\left(\widehat{\mathbf{X}}_{k}^{-}, \overline{\mathbf{V}}_{k}\right)+C_{k}\left(\mathbf{X}_{k}-\widehat{\mathbf{X}}_{k}^{-}\right)+D_{k}\left(\mathbf{V}_{k}-\overline{\mathbf{V}}_{k}\right)
\end{aligned}
$$

where

$$
\begin{aligned}
A_{k} & =\left.\frac{\partial F_{k}}{\partial \mathbf{X} M_{k-1}}\right|_{X_{k-1}=\widehat{X}_{k-1}, W_{\mathrm{k}}=\bar{W}_{k}} \\
B_{k} & =\left.\frac{\partial F_{k}}{\partial \mathbf{W}_{k}}\right|_{X_{k-1}=\widehat{X}_{k-1}, W_{\mathrm{k}}=\bar{W}_{k}} \\
C_{k} & =\left.\frac{\partial G_{k}}{\partial \mathbf{X}_{k}}\right|_{X_{k}=\widehat{X}_{k}^{-}, V_{k}=\bar{V}_{k}} \\
D_{k} & =\left.\frac{\partial G_{k}}{\partial V_{k}}\right|_{X_{k}=\widehat{X}_{k}^{-}, V_{k}=\bar{V}_{k}}
\end{aligned}
$$

With these notations, the EKF algorithm may be summarized as follows [6]:

$$
\begin{aligned}
\widehat{\mathbf{X}}_{k}^{-} & =F_{k}\left(\widehat{\mathbf{X}}_{k-1}, \overline{\boldsymbol{W}}_{k}\right) \\
\boldsymbol{I}_{k} & =\boldsymbol{Y}_{k}-G_{k}\left(\widehat{\mathbf{X}}_{k}^{-}, \overline{\boldsymbol{V}}_{k}\right) \\
P_{k}^{-} & =A_{k} P_{k-1} A_{k}^{T}+B_{k} Q_{k} B_{k}^{T} \\
K_{k} & =P_{k}^{-} C_{k}^{T}\left(C_{k} P_{k}^{-} C_{k}^{T}+D_{k} R_{k} D_{k}^{T}\right)^{-1} \\
P_{k} & =P_{k}^{-}-K_{k} C_{k} P_{k}^{-} \\
\widehat{\mathbf{X}}_{k} & =\widehat{\mathbf{X}}_{k}^{-}+K_{k} \boldsymbol{I}_{k}
\end{aligned}
$$

where $\mathbf{X}_{k}^{-}=E\left\{\widehat{\mathbf{X}}_{k} \mid \mathbf{Y}_{0}, \ldots, \mathbf{Y}_{k-1}\right\} \quad$ is the a priori estimation of $\mathbf{X}_{k}$ which uses all the observations till time instant $k-1$ to estimate the $\mathbf{X}_{k} . \mathbf{I}_{k}$ is the innovation vector, $P_{k}^{-}=E\left\{\left(\boldsymbol{X}_{k}-\widehat{\mathbf{X}}_{k}^{-}\right)\left(\boldsymbol{X}_{k}-\widehat{\mathbf{X}}_{k}^{-}\right)^{T}\right\} \quad$ and $P_{k}=E\left\{\left(\boldsymbol{X}_{k}-\mathbf{X}_{k}\right)\left(\widehat{\boldsymbol{X}}_{k}-\widehat{\mathbf{X}}_{k}\right)^{T}\right\}$ are respectively the a priori and the a posteriori estimation of the state vector covariance matrix, and $\widehat{\mathbf{X}}_{k}=E\left\{\boldsymbol{X}_{k} \mid \boldsymbol{Y}_{0}, \ldots, \boldsymbol{Y}_{k}\right\}$ is the a posteriori estimation of $\widehat{\mathbf{X}}_{k}$. By knowing the initial estimate of the state vector $\widehat{\mathbf{X}}_{0}$ and state covariance matrix $P_{0}$, estimation of the state variables through the recursive procedure would be possible.

\subsubsection{Extended Kalman smoother}

The Kalman smoother (KS) estimates the state of a system at time instant $k$ using the observations before and after $k$. As with the EKF, the extended Kalman smoother (EKS) is the extension of KS to nonlinear systems. The performance of the EKS is generally superior to the EKF, since it uses additional observations for its estimate. The EKS algorithm essentially consists of a forward EKF algorithm up to each time instant $k$, combined with a backward recursive smoothing algorithm using the observations beyond time instant $k$. Smoothing algorithms are usually divided into three types: fixed-interval, fixed-lag, and fixed-point [6]. Fixed-interval smoothers use all the observations over a fixed interval to estimate the state of a system at all times in the same interval and are most common used for off-line processing. In this paper, the fixed-interval EKS is used, since the filtering procedure is performed off-line on the whole length of each PCG signal. 


\section{Proposed phonocardiogram model}

The cardiovascular system has a quasi-periodic nature, which arises from quasi-periodic activity of cardiac electrical system. In other words, the cardiac electrical system sends electrical pulses alternatively which stimulate the heart and cause it to contract. The quasi-periodic nature of the heart makes its output signals quasi-periodic too. However, this quasi-periodicity means that cardiac signals follow a semi-deterministic pattern in each heartbeat, while some elements (or parameters) of this pattern may vary from beat to beat, which reflect the stochastic characteristics of heart signals. As an illustration, in McSharry's model for ECG signal, the semi-deterministic pattern is the sum of five Gaussian kernels as

$z=\sum_{i \in\{P, Q, R, S, T\}} \alpha_{i} \exp \left(-\frac{\Delta \theta_{i}^{2}}{2 b_{i}^{2}}\right)$

where the kernels' parameters model the stochastic characteristics of the ECG in each beat and are assumed to be random variables whose mean values are shown in Table 1.

In the recent years, some attempts have been made to model the two dominant components of the PCG, namely S1 and S2. Auto-Regressive Moving-Average (ARMA) models and higher order spectra analyses were then explored $[7,8]$. The main drawback of such statistical models is that they ignore the quasiperiodic morphology of the signal. Mathematical models based on signal morphology for representing the time and frequency characteristics of the PCG were also investigated [9-11]. But they on the other hand ignore the stochastic characteristics of the signal. Furthermore, in some other works, Matching Pursuit (MP) algorithm was used for analysis and synthesis of the phonocardiogram, but no explicit models were proposed [12-15]. Therefore, lack of a dynamical model for generating artificial PCG signals is easily felt.

\subsection{Phonocardiogram morphology}

A PCG beat mainly consists of two distinct sounds, $\mathrm{S} 1$ and S2, sample waveforms of which are depicted in Fig. 1. Waveforms of heart sounds vary from subject to subject and from beat to beat in amplitude, number of peaks and troughs, and spread in time. With all these differences, still there are some analogies among all waveforms. A closer look at the waveforms suggests the idea of damped sinusoids, since the frequency of vibration is almost constant along their waveforms. On the other hand, several Gaussian functions can model their envelope shapes. Hence, based on PCG morphology, it seems that Gabor kernels may be an appropriate choice for modeling heart sounds waveform. The idea of using Gabor kernels for modeling heart sounds is quite consistent with the previous works done on analysis and synthesis of the heart sounds using Matching Pursuit algorithm over a dictionary consisting of Gabor atoms [12-15]. As a result, one heartbeat of PCG signal, including S1 and S2, can be modeled as follows:

$z(\theta)=S 1(\theta)+S 2(\theta)$

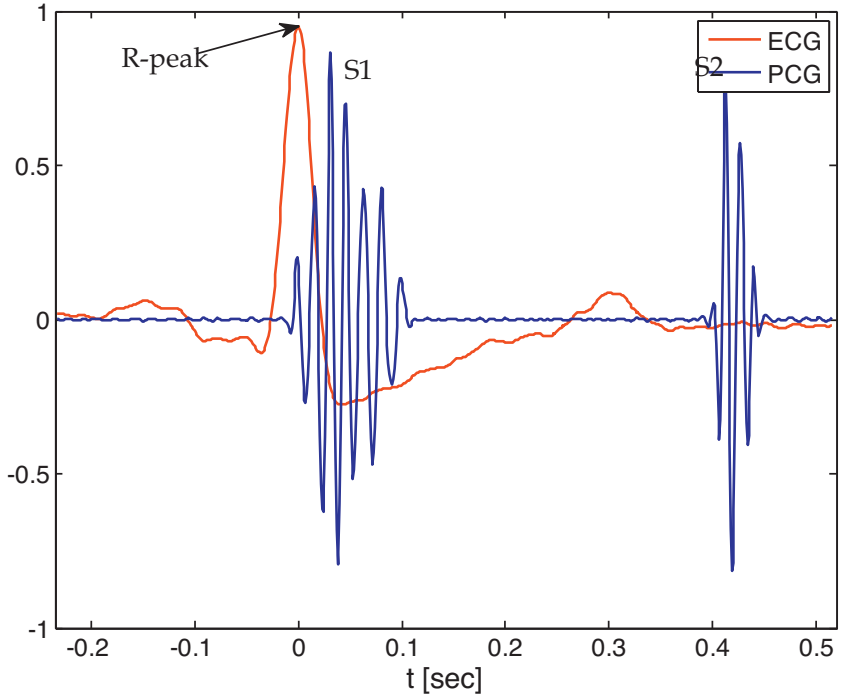

Fig. 1. A phonocardiogram signal from a healthy person, including the first heart sound ( $\mathrm{S} 1)$ and the second heart sound $(\mathrm{S} 2)$. The time is specified relative to the position of the R-peak in the synchronous electrocardiogram (ECG).

and

$S 1(\theta)=\sum_{i=1}^{m} \alpha_{i} \exp \left(-\frac{\left(\theta-\mu_{i}\right)^{2}}{2 \sigma_{i}^{2}}\right) \cos \left(f_{i} \theta-\varphi_{i}\right)$
$S 2(\theta)=\sum_{i=1}^{n} \alpha_{i} \exp \left(-\frac{\left(\theta-\mu_{i}\right)^{2}}{2 \sigma_{i}^{2}}\right) \cos \left(f_{i} \theta-\varphi_{i}\right)$

where $\alpha_{i}, \mu_{i}$, and $\sigma_{i}$ are the amplitude, center and width parameters of the Gaussian terms, and $f_{i}, \varphi_{i}$ are frequency and phase shift of the sinusoid terms, respectively, and $m$ and $n$ indicate the number of Gabor kernels used for modeling each heart sound ( $m$ kernels for S1 and $n$ kernels for S2). The model parameters are random variables with specific probability density function. In other words, these random variables model the stochastic characteristics of each PCG beat. $\theta$ is the phase parameter in radians which indicates the relative time-difference of the events in a PCG beat with respect to a fixed reference point. Such a reference point must be easily detectable in each heartbeat. Since finding such a point in the PCG needs very complicated processing techniques, an external reference point may be used. As it can be seen from Fig. 1, there is a close relationship between events in ECG and PCG. For example, the S1 occurs slightly after the ECG R-peak. Hence, the R-peak in the simultaneously recorded ECG signal can be considered as an external reference point. On the other hand, to extract heartbeats from the PCG, we set down the criterion that the middle point of each R-to$\mathrm{R}$ distances is considered as the ending point of the previous beat and starting point of next beat (as illustrated in Fig. 4); so PCG beats become distinguishable. Therefore, the phase signal $\theta$ for each PCG beat would be the same as the phase signal defined in [1]. Thus, $\theta$ ranges in $[-\pi, \pi]$ and as it is a function of time (since heartbeat duration may vary in time), $z$ can also be seen as a time-dependent function. This issue is employed in the introduced dynamical model for the PCG in next section. The $\mu_{i}, \sigma_{i}$ and $\varphi_{i}$ parameters are in radians, while the $f_{i}$ is without 
Table 2

Mean values of the phonocardiogram (PCG) model parameters.

\begin{tabular}{|c|c|c|c|c|}
\hline \multirow{2}{*}{$\frac{\text { Heart sound }}{\text { Index (i) }}$} & \multicolumn{2}{|l|}{$\mathrm{S} 1$} & \multicolumn{2}{|l|}{$\mathrm{S} 2$} \\
\hline & 1 & 2 & 1 & 2 \\
\hline$\alpha_{i}$ & 0.4250 & 0.6875 & 0.5575 & 0.4775 \\
\hline$\mu_{i}$ (radians) & $\pi / 12$ & $3 \pi / 19$ & $3 \pi / 4$ & $7 \pi / 9$ \\
\hline$\sigma_{i}($ radians $)$ & 0.1090 & 0.0816 & 0.0723 & 0.1060 \\
\hline$f_{i}$ & 65.87 & 74.61 & 71.10 & 68.37 \\
\hline$\varphi_{i}$ (radians) & $3 \pi / 4$ & $9 \pi / 11$ & $7 \pi / 8$ & $3 \pi / 4$ \\
\hline
\end{tabular}

dimension. In fact, $f_{i}$ is the frequency coefficient of each Gabor kernel; by multiplying $f_{i}$ by $f_{m}$, the actual frequency of each kernel (in Hertz) is obtainable where $f_{m}$ is the heart rate in Hertz.

\subsection{Phonocardiogram dynamical model}

Like McSharry's model for ECG, the proposed dynamical model for PCG generates a 3-D trajectory whose projection on the $(x, y)$ plane moves around a limit cycle of unit radius. Each revolution on this circle corresponds to one heartbeat. Waveform of phonocardiogram signal is produced using the motion of the trajectory in the $z$ direction. The dynamical equations of the trajectory of the model are given by a set of three ordinary differential equations,

$\dot{r}=r(1-r)$

$\dot{\theta}=\omega$

$\dot{z}=\frac{\partial S 1}{\partial t}+\frac{\partial S 2}{\partial t}=-\sum_{i=1}^{m+n}\left(\omega \frac{\alpha_{i}}{\sigma_{i}^{2}}\left(\theta-\mu_{i}\right) \exp \left(-\frac{\left(\theta-\mu_{i}\right)^{2}}{2 \sigma_{i}^{2}}\right)\right.$

where $\theta=\operatorname{atan} 2(y, x)$ is the four quadrant arctangent of the real parts of the elements of $x$ and $y$, with $-\pi \leq \theta \leq \pi$, and can be interpreted as the phase signal defined in previous section. $\omega$ is the angular velocity of the trajectory as it moves around the limit cycle and is related to beat-to-beat heart rate by $\omega(t)=\frac{2 \pi}{R(t)}$ where $R(t)$ represents the time series generated by the RRintervals using the synchronous ECG signal. Considering $\omega$ constant, and integrating the third equation in (12), we reach to equation (10). However, in the condition of $\omega$ being variable in time, the equations of motion given by (12) are integrated numerically using a fourth-order Runge-Kutta method with a fixed time step $\Delta t=1 / f_{s}$ where $f_{s}$ is the sampling frequency of the synthetic PCG signal. Thus, both beat-to-beat heart rate (HR) and sampling frequency of the synthetic PCG signal can be adjusted.

The model parameters of (12) for a PCG signal can be easily achieved by fitting (11) to signal heartbeats. All parameters except $\mu_{i}$ are independent from the synchronous ECG signal. The $\mu_{i}$ parameters indicate the location of Gabor kernels with respect to the R-peak in the synchronous ECG.

The PCG, in contrast to ECG, is less quasi-periodic and variation of PCG model parameters from beat to beat is significant. However, to give a visual perception of the model parameters, Table 2 shows their mean values, which are achieved by the

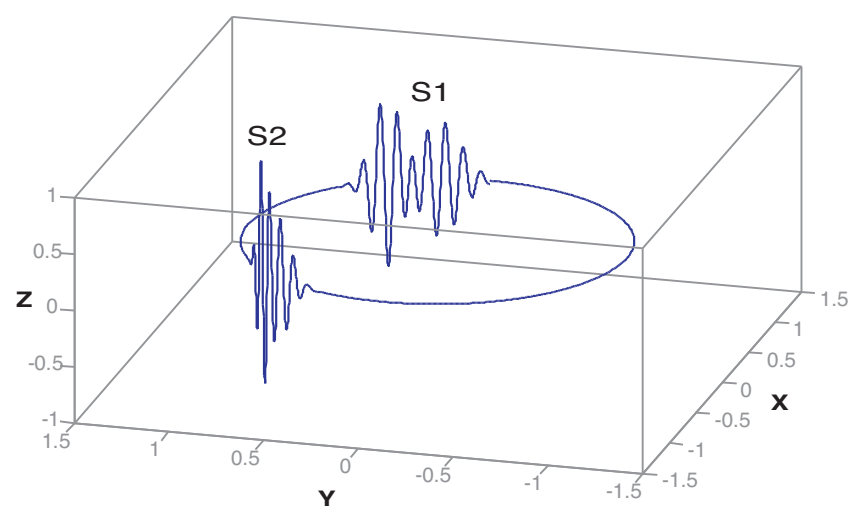

Fig. 2. Typical trajectory generated by the dynamical model (11) in the 3-D space given by $(\mathrm{x}, \mathrm{y}, \mathrm{z})$. Each entire movement around this trajectory is equivalent to one heartbeat. The model parameters are selected based on a real phonocardiogram (PCG) beat.

analysis of normalized PCG of a healthy subject, where each heart sound is modeled with two Gabor kernels.

A trajectory generated by (12) in three dimensions is depicted in Fig. 2. The $z$ variable of this trajectory, when plotted versus time, represents the synthetic PCG signal with realistic morphology.

\section{Model-based phonocardiogram denoising framework}

In the conventional denoising approaches, we usually look for a technique that removes (or reduces) the effect of the

$\left.\cos \left(f_{i} \theta-\varphi_{i}\right)+\alpha_{i} f_{i} \exp \left(-\frac{\left(\theta-\mu_{i}\right)^{2}}{2 \sigma_{i}^{2}}\right) \sin \left(f_{i} \theta-\varphi_{i}\right)\right)$

noise contaminating the desired signal. But from a statistical signal-processing viewpoint, denoising may be considered as an estimation approach of the desired signal from its noisy version. As described before, the KF is one such method and may be used if the underlying dynamic model of the system is known. In order to do so, we intend to use the PCG dynamical model introduced in Section 3.2 within a KF framework.

The main concept of the proposed Bayesian denoising framework for PCG is illustrated in Fig. 3. In this framework, the PCG and the phase signal are considered as the states of a system whose underlying dynamic model is provided by the PCG dynamical model. This section concerns the details of the proposed denoising framework.

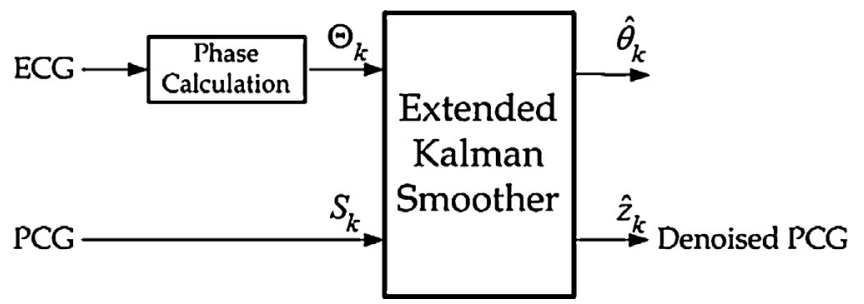

Fig. 3. General block diagram of the proposed model-based Bayesian phonocardiogram (PCG) denoising. 


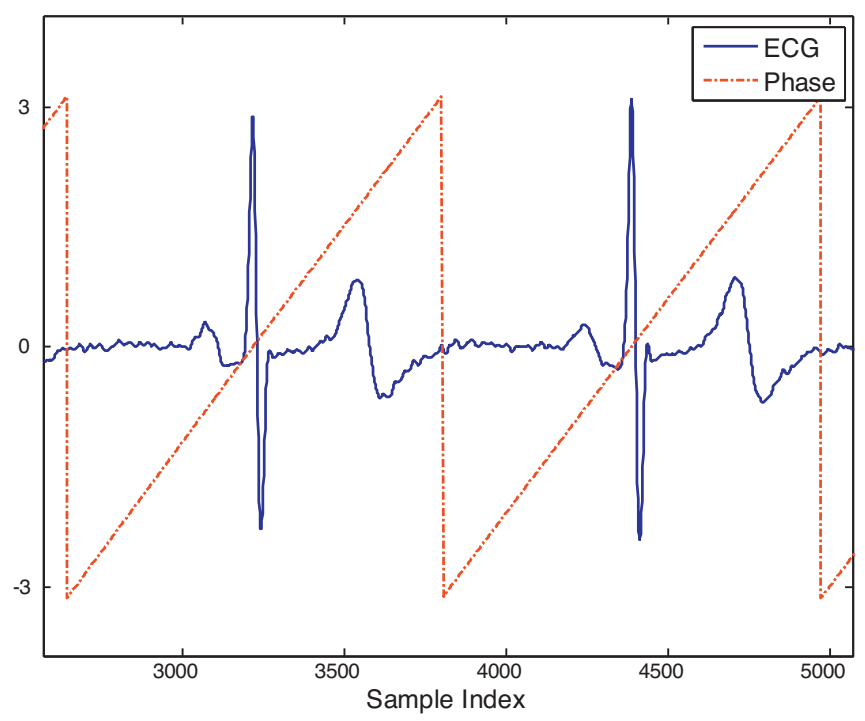

Fig. 4. Phase signal obtained from the synchronous electrocardiogram (ECG) signal.

\subsection{Combining the proposed dynamical model with the Kalman filter structure}

Assuming a small sampling period of $\delta$, we can discretize the equations of (12) as

$\dot{\theta}=\frac{\theta_{k+1}-\theta_{k}}{\delta}$

$\dot{z}=\frac{z_{k+1}-z_{k}}{\delta}$

Therefore, the discrete version of introduced dynamical model in (12) is as follows:

$\theta_{k+1}=\theta_{k}+\delta \omega_{k}$

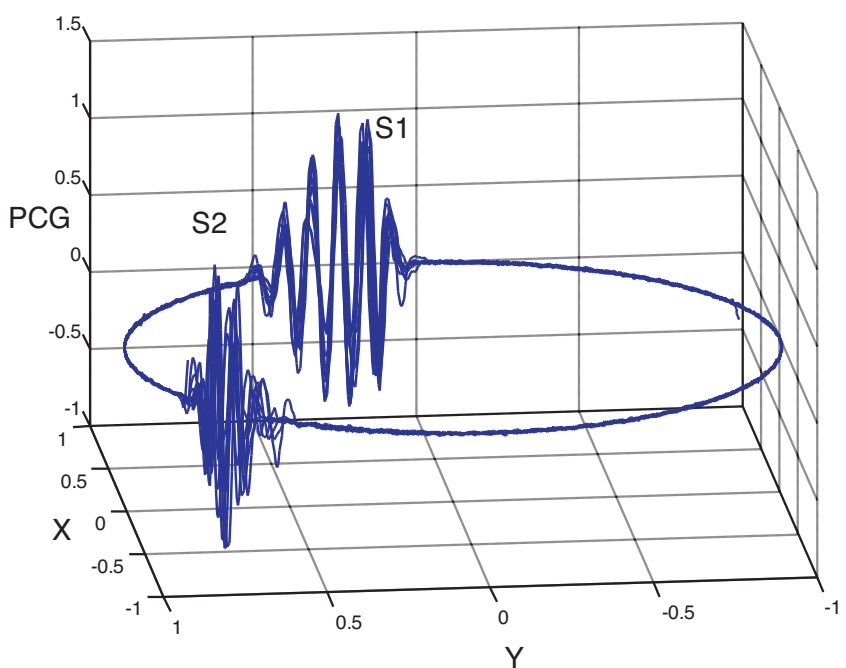

Fig. 5. A phonocardiogram (PCG) signal plotted in cylindrical coordinates with corresponding phase signal.

where $l=m+n$ is the total number of Gabor kernels used to model the heart sounds, and the process noise covariance matrix is $Q_{k}=E\left\{\boldsymbol{W}_{k} \boldsymbol{W}_{k}^{T}\right\}-\overline{\boldsymbol{W}}_{k} \overline{\boldsymbol{W}}_{k}^{T}$. The process noises are considered to be uncorrelated and as a result the covariance matrix of the process noise is diagonal.

\subsection{Linearization of the phonocardiogram dynamical model}

Since the state equations are nonlinear, extended Kalman filter should be used. In order to set up an EKF, it is necessary to

$z_{k+1}=z_{k}-\delta \omega_{k} \sum_{i}\left(\frac{\alpha_{i}}{\sigma_{i}^{2}}\left(\theta_{k}-\mu_{i}\right) \exp \left(-\frac{\left(\theta_{k}-\mu_{i}\right)^{2}}{2 \sigma_{i}^{2}}\right) \cos \left(f_{i} \theta_{k}-\varphi_{i}\right)+\alpha_{i} f_{i} \exp \left(-\frac{\left(\theta_{k}-\mu_{i}\right)^{2}}{2 \sigma_{i}^{2}}\right) \sin \left(f_{i} \theta_{k}-\varphi_{i}\right)\right)+\eta_{k}$

where $\eta_{k}$ is a random additive noise that models the uncertainty of the dynamic model. The $\alpha_{i}, \mu_{i}, \sigma_{i}, f_{i}, \varphi_{i}$ and $\omega$ are random variables in each beat, which can be assumed as i.i.d. Gaussian random variables, and as Gaussian random processes in time. Henceforth, to make the model fit into Kalman filter framework, we assume $\theta_{k}$ and $z_{k}$ as state variables, and the $\alpha_{i}, \mu_{i}, \sigma_{i}, f_{i}, \varphi_{i}$, $\omega_{k}$ and $\eta_{k}$ as process noises. Thus, the state variables and process noise vector are defined as follows:

$$
\begin{aligned}
\boldsymbol{X}_{k} & =\left[\begin{array}{ll}
\theta_{k} & z_{k}
\end{array}\right]^{T} \\
\boldsymbol{W}_{k} & =\left[\begin{array}{llllll}
\alpha_{1} \ldots \alpha_{l} & \mu_{1} \ldots \mu_{l} & \sigma_{1} \ldots \sigma_{l} & f_{1} \ldots f_{l} & \varphi_{1} \ldots \varphi_{l} & \omega_{k} \ldots \eta_{k}
\end{array}\right]
\end{aligned}
$$

$$
\begin{aligned}
\frac{\partial F_{1_{k}}}{\partial z_{k}}=0 \quad, \quad \frac{\partial F_{1_{k}}}{\partial \theta_{k}}=\frac{\partial F_{2_{k}}}{\partial z_{k}}=1 & \\
\frac{\partial F_{2_{k}}}{\partial \theta_{k}}= & -\delta \omega_{k} \sum_{i}\left(\frac{\alpha_{i}}{\sigma_{i}^{2}}\left(1-\frac{\left(\theta_{k}-\mu_{i}\right)^{2}}{\sigma_{i}^{2}}\right) \exp \left(-\frac{\left(\theta_{k}-\mu_{i}\right)^{2}}{2 \sigma_{i}^{2}}\right) \cos \left(f_{i} \theta_{k}-\varphi_{i}\right)-\frac{\alpha_{i} f_{i}}{\sigma_{i}^{2}}\left(\theta_{k}-\mu_{i}\right) \exp \left(-\frac{\left(\theta_{k}-\mu_{i}\right)^{2}}{2 \sigma_{i}^{2}}\right) \sin \left(f_{i} \theta_{k}-\varphi_{i}\right)\right. \\
& \left.+\alpha_{i} f_{i}^{2} \exp \left(-\frac{\left(\theta_{k}-\mu_{i}\right)^{2}}{2 \sigma_{i}^{2}}\right) \cos \left(f_{i} \theta_{k}-\varphi_{i}\right)\right)
\end{aligned}
$$

linearize the nonlinear state equations. So by defining $F_{1_{k}}$ and $F_{2_{k}}$ as:

$$
\begin{aligned}
& \theta_{k+1}=F_{1_{k}}\left(\theta_{k}, \omega_{k}\right) \\
& z_{k+1}=F_{2_{k}}\left(z_{k}, \theta_{k}, \alpha_{i}, \mu_{i}, \sigma_{i}, f_{i}, \varphi_{i}, \omega_{k}, \eta_{k}\right)
\end{aligned}
$$

the following equations represent the linearized model with respect to the state variables $\theta_{k}$ and $z_{k}$ : 
Similarly, the linearized versions of (16) with respect to process noises are as

$$
\begin{aligned}
& \frac{\partial F_{1_{k}}}{\partial \alpha_{i}}=\frac{\partial F_{1_{k}}}{\partial \mu_{i}}=\frac{\partial F_{1_{k}}}{\partial \sigma_{i}}=\frac{\partial F_{1_{k}}}{\partial f_{i}}=\frac{\partial F_{1_{k}}}{\partial \varphi_{i}}=\frac{\partial F_{1_{k}}}{\partial \eta_{k}}=0 \quad, \quad \frac{\partial F_{1_{k}}}{\partial \omega_{k}}=\delta \\
& \frac{\partial F_{2_{k}}}{\partial \alpha_{i}}=-\delta \omega_{k}\left(\frac{\left(\theta_{k}-\mu_{i}\right)}{\sigma_{i}^{2}} \exp \left(-\frac{\left(\theta_{k}-\mu_{i}\right)^{2}}{2 \sigma_{i}^{2}}\right) \cos \left(f_{i} \theta_{k}-\varphi_{i}\right)+f_{i} \exp \left(-\frac{\left(\theta_{k}-\mu_{i}\right)^{2}}{2 \sigma_{i}^{2}}\right) \sin \left(f_{i} \theta_{k}-\varphi_{i}\right)\right) \\
& \frac{\partial F_{2_{k}}}{\partial \mu_{i}}=-\delta \omega_{k}\left(\frac{\alpha_{i}}{\sigma_{i}^{2}}\left(1-\frac{\left(\theta_{k}-\mu_{i}\right)^{2}}{\sigma_{i}^{2}}\right) \exp \left(-\frac{\left(\theta_{k}-\mu_{i}\right)^{2}}{2 \sigma_{i}^{2}}\right) \cos \left(f_{i} \theta_{k}-\varphi_{i}\right)-\frac{\alpha_{i} f_{i}}{\sigma_{i}^{2}}\left(\theta_{k}-\mu_{i}\right) \exp \left(-\frac{\left(\theta_{k}-\mu_{i}\right)^{2}}{2 \sigma_{i}^{2}}\right) \sin \left(f_{i} \theta_{k}-\varphi_{i}\right)\right) \\
& \frac{\partial F_{2_{k}}}{\partial \sigma_{i}}=2 \delta \omega_{k} \frac{\alpha_{i}}{\sigma_{i}^{3}}\left(\theta_{k}-\mu_{i}\right) \exp \left(-\frac{\left(\theta_{k}-\mu_{i}\right)^{2}}{2 \sigma_{i}^{2}}\right)\left(\left(1-\frac{\left(\theta_{k}-\mu_{i}\right)^{2}}{2 \sigma_{i}^{2}}\right) \cos \left(f_{i} \theta_{k}-\varphi_{i}\right)-\frac{1}{2} f_{i}\left(\theta_{k}-\mu_{i}\right) \sin \left(f_{i} \theta_{k}-\varphi_{i}\right)\right) \\
& \frac{\partial F_{2_{k}}}{\partial f_{i}}=\delta \omega_{k} \alpha_{i}\left(\frac{\theta_{k}}{2 \sigma_{i}^{2}}\left(\theta_{k}-\mu_{i}\right)-1\right) \exp \left(-\frac{\left(\theta_{k}-\mu_{i}\right)^{2}}{2 \sigma_{i}^{2}}\right) \sin \left(f_{i} \theta_{k}-\varphi_{i}\right)-\delta \omega_{k} \alpha_{i} f_{i} \theta_{k} \exp \left(-\frac{\left(\theta_{k}-\mu_{i}\right)^{2}}{2 \sigma_{i}^{2}}\right) \cos \left(f_{i} \theta_{k}-\varphi_{i}\right) \\
& \frac{\partial F_{2_{k}}}{\partial \varphi_{i}}=\delta \omega_{k} \alpha_{i} f_{i} \exp \left(-\frac{\left(\theta_{k}-\mu_{i}\right)^{2}}{2 \sigma_{i}^{2}}\right) \cos \left(f_{i} \theta_{k}-\varphi_{i}\right)-\frac{\delta \omega_{k} \alpha_{i}}{\sigma_{i}^{2}}\left(\theta_{k}-\mu_{i}\right) \exp \left(-\frac{\left(\theta_{k}-\mu_{i}\right)^{2}}{2 \sigma_{i}^{2}}\right) \sin \left(f_{i} \theta_{k}-\varphi_{i}\right) \\
& \frac{\partial F_{2_{k}}}{\partial \omega_{k}}=-\delta \sum_{i}\left(\frac{\alpha_{i}}{\sigma_{i}^{2}}\left(\theta_{k}-\mu_{i}\right) \exp \left(-\frac{\left(\theta_{k}-\mu_{i}\right)^{2}}{2 \sigma_{i}^{2}}\right) \cos \left(f_{i} \theta_{k}-\varphi_{i}\right)+\alpha_{i} f_{i} \exp \left(-\frac{\left(\theta_{k}-\mu_{i}\right)^{2}}{2 \sigma_{i}^{2}}\right) \sin \left(f_{i} \theta_{k}-\varphi_{i}\right)\right) \\
& \frac{\partial F_{2_{k}}}{\partial \eta_{k}}=1 \quad, \quad i \in\left\{S 1^{1: m}, S 2^{1: n}\right\}
\end{aligned}
$$

\subsection{Observation equations}

There are two state variables in the proposed framework: the PCG $z_{k}$, and the phase $\theta_{k}$. The noisy PCG, which is recorded and is available may be considered as observation for the PCG $z_{k}$, and can be written as $S_{k}=z_{k}+v_{2_{k}}$ where $v_{2_{k}}$ is the noise of PCG signal. However, we look for another observation for the state variable $\theta_{k}$. This issue is completely studied in [2] and can be extended to our framework where the phase signal $\theta_{k}$ can be obtained from the associated ECG signal. As explained in Section 3.1, the R-peak may be assumed to be an external reference point for the heart sounds and hence is located at $\theta=0$. Accordingly, the ECG contents lying between two consecutive R-peaks are assumed to have a phase between $-\pi$ and $\pi$, as illustrated in Fig. 4. So, by simply detecting the R-peaks in the synchronous ECG, the observation for the phase signal $\theta_{k}$ is achieved [2]. This linear approximation of phase is considered as phase observation and can be written as: $\Theta_{k}=\theta_{k}+v_{1_{k}}$ where $v_{1_{k}}$ is the noise of the phase signal.

Hence, the noisy PCG and the phase observation can be related to the state vector as follows:

$\boldsymbol{Y}_{k}=\left[\begin{array}{ll}1 & 0 \\ 0 & 1\end{array}\right] \boldsymbol{X}_{k}+\boldsymbol{V}_{k}$

where $\quad \boldsymbol{Y}_{k}=\left[\begin{array}{ll}\Theta_{k} & S_{k}\end{array}\right]^{T}, \quad \boldsymbol{V}_{k}=\left[\begin{array}{ll}v_{1_{k}} & v_{2_{k}}\end{array}\right]^{T} . \quad R_{k}=$ $E\left\{\boldsymbol{V}_{k} \boldsymbol{V}_{k}^{T}\right\}-\overline{\boldsymbol{V}}_{k} \overline{\boldsymbol{V}}_{k}^{T}$ is the covariance matrix of the observation noise vector. Although the noises contaminating PCG may be generally nonlinear and non-Gaussian, but for simplicity in this study, they all are assumed to be an additive white Gaussian noise i.e. $v_{2_{k}}$. The $v_{1_{k}}$ and $v_{2_{k}}$ are considered to be uncorrelated and therefore the covariance matrix of the observation noise is diagonal.

\subsection{Denoising procedure}

As illustrated in Fig. 3, in order to denoise PCG signal, the discrete version of dynamical model in (14) is used within a Kalman filter structure. The PCG and phase signals are assumed as state variables, which are estimated from their observations, namely noisy PCG and approximated phase signal calculated from synchronous ECG.

\subsection{Estimation of the Kalman filter parameters}

For the implementation of the filter, the values of the measurement and process noise covariance matrices should be known. As we use $l$ Gabor kernels in [12] for modeling a PCG beat, consequently the process noise vector defined in (15) has $5 l+2$ elements, and the covariance matrix of the process noise, $Q_{k}$, has a dimension of $(5 l+2) \times(5 l+2)$. Nevertheless, assumption of uncorrelated noise sources, which here is a reasonable assumption, may lead the $Q_{k}$ matrix to be diagonal, similar to $R_{k}$. It is more convenient to automate the parameter estimation from the signal itself since these parameters should be adjusted for each input PCG signal. As described before, each PCG beat can be plotted in cylindrical coordinates $(r, \theta, z)$, where $r=1, \theta$ and $z$ equal to phase and PCG signals, respectively. A phase-wrapped PCG of several beats obtained by this approach is illustrated in Fig. 5. The mean and variance of this phase-wrapped PCG may be calculated for all phases between $-\pi$ and $\pi$ (or 0 and $2 \pi$ ). This gives the average of the PCG waveform, including $\mathrm{S} 1$ and S2. The mean and standard deviation of the same PCG signal depicted in Fig. 5 is shown in Fig. 6. Now, it is possible to estimate the model parameters for the given PCG signal. Here the problem is to find the optimal parameters of (12) that can best model the mean PCG. There are many optimization 


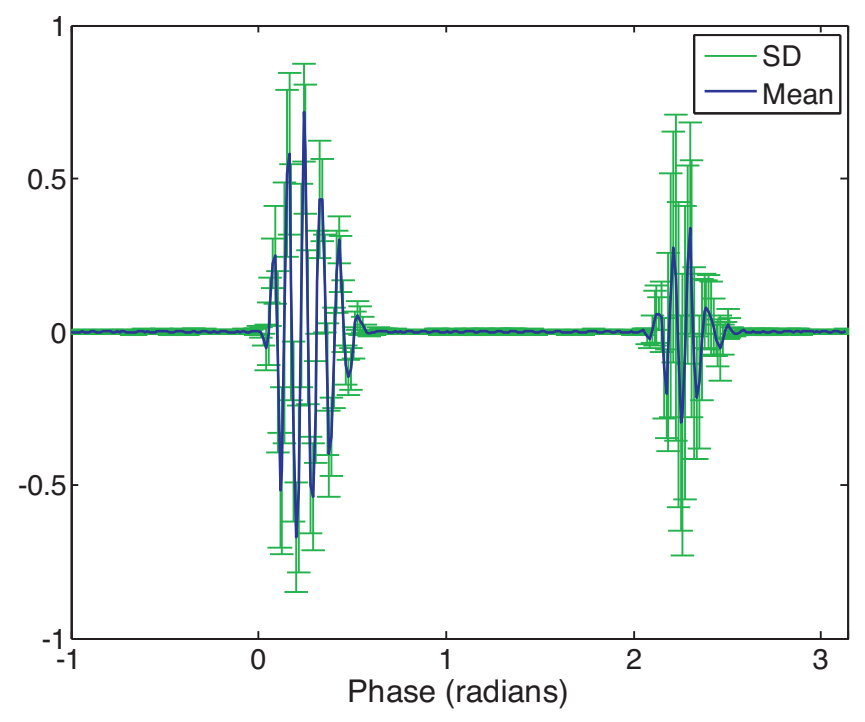

Fig. 6. The mean and standard deviation-bar of a 10-beat-phonocardiogram (PCG) signal. The standard deviation-bar features the beat-to-beat variations in a PCG signal.

methods that can be used for this purpose. In this case, the lsqnonlin function of MATLAB ${ }^{\circledR}$ was used to find the model parameters of the Gabor kernels.

An estimation for the variances of the process noises (except $\omega_{k}$ and $\eta_{k}$ ) may be done using the standard deviation of the phase-wrapped PCG. In fact, the deviation of the parameters of the Gabor kernels in (12) around the estimated mean should be calculated. Again, a nonlinear least-square optimization method should be used to find the optimal parameters that generate the best model of the mean PCG within the upper and lower ranges of $\overline{P C G}(\theta)+\sigma_{P C G}(\theta)$ and $\overline{P C G}(\theta)-\sigma_{P C G}(\theta)$.

As discussed in Section 3.2, the angular frequency $\omega$ may be related to beat-to-beat heart rate as $\omega=\frac{2 \pi}{R(t)}$, where $R(t)$ is the RR-series in ECG signal. Therefore, mean and standard deviation of $\omega$ can be easily calculated using the RR-intervals of the whole ECG signal (in on-line processing estimation of the mean and SD of the parameter $\omega$ may be obtained from the first portions of ECG signal). As said before, $\eta_{k}$ models the uncertainty of the dynamical model, which is assumed as a white Gaussian noise with appropriate variance.

The measurement noise of the phase signal $v_{1_{k}}$ arises from sampling error whose interpretation is quite studied in [2]. According to it, $v_{1_{k}}$ would be uniformly distributed in the range of $[-\omega \delta / 2,+\omega \delta / 2]$, where $\delta$ is the sampling period, and as a result $E\left\{v_{1_{k}}^{2}\right\}=(\omega \delta)^{2} / 12$.

There are some methods to estimate the variance of the measurement noise, $v_{2_{k}}$. One way is to calculate the noise power from the deviation of the whole signal around the mean PCG, or from the portions of PCG signal where there are no events, namely $\mathrm{S} 1$ and $\mathrm{S} 2$.

\section{Simulation results and discussions}

The proposed PCG dynamical model and model-based PCG Bayesian denoising framework were finally implemented in
MATLAB $^{\circledR}$. All the PCG and ECG signals used in this study were normalized and the results presented next are based on this issue.

\subsection{Dataset}

PCG signals are provided by Prof. Louis-Gilles Durand of the Institut de Recherche Clinique de Montréal (IRCM), Quebec, Canada. The data were recorded on healthy subjects at the pulmonary area (2nd and 4th left intercostal space along the sternal border) and at the apex on the chest wall, using two types of microphones (BSM, Andromed). Four series of recordings were performed:

- BSM1 at 2nd LICS and BSM2 at the apex;

- BSM1 at 2nd LICS and BSM2 at 4th LICS;

- microphone 1 at 2nd LICS and microphone 2 at the apex;

- microphone 1 at 2nd LICS and microphone 2 at 4th LICS.

Heart sounds and electrocardiogram were recorded simultaneously. The sampling rates of ECG and PCG are $200 \mathrm{~Hz}$ and $2000 \mathrm{~Hz}$, respectively; in this study both signals are resampled to $1000 \mathrm{~Hz}$.

\subsection{Synthetic phonocardiogram generator}

As mentioned in Section 3.2, PCG model parameters may vary from beat to beat, and also person to person. To have an understanding of the valid model parameters for PCG, a comprehensive investigation should be done. In this study, the synthetic heart sounds are generated using the model parameters obtained from analysis of PCGs of some healthy people. Fig. 7 illustrates four different morphologies of synthetic heart sound waveforms for one heartbeat. Each heart sound is generated by using two Gabor kernels whose parameters are listed in Table 3. Observational uncertainty is also considered in Fig. 8 by adding white Gaussian noise to the synthetic signal, yielding a similar signal to a section of real PCG from a normal human.

Table 3

The values of $\left(\alpha_{i}, \mu_{i}, \sigma_{i}, f_{i}, \varphi_{i}\right)$ of the Gabor kernels used in the phonocardiogram (PCG) dynamical model, to generate the heart sound waveforms depicted in Fig. 7 respectively, corresponding to (a), (b), (c) and (d) subfigures. Each heart sound is generated by using two kernels.

\begin{tabular}{lll}
\hline Figure Index & \multicolumn{2}{l}{ Gabor Kernels } \\
\cline { 2 - 3 } & S1 & S2 \\
\hline (a) & $(0.80,0.26,0.08,66.66,2.77)$ & $(0.08,2.41,0.06,66.92,3.14)$ \\
& $(0.80,0.45,0.08,78.85,1.73)$ & $(0.90,2.44,0.08,71.19,3.14)$ \\
(b) & $(0.45,0.28,0.14,67.86,2.83)$ & $(0.70,2.31,0.05,69.12,3.14)$ \\
& $(0.93,0.47,0.07,76.59,3.14)$ & $(0.51,2.46,0.07,73.76,0.00)$ \\
(c) & $(0.33,0.23,0.08,63.21,2.00)$ & $(0.53,2.39,0.08,62.83,0.18)$ \\
& $(0.80,0.44,0.07,68.05,3.14)$ & $(0.52,2.46,0.06,62.27,3.14)$ \\
(d) & $(0.43,0.25,0.11,65.97,2.23)$ & $(0.43,2.36,0.04,67.36,3.14)$ \\
& $(0.76,0.50,0.08,71.94,3.14)$ & $(0.33,2.45,0.17,66.98,3.13)$ \\
\hline & &
\end{tabular}


(a)

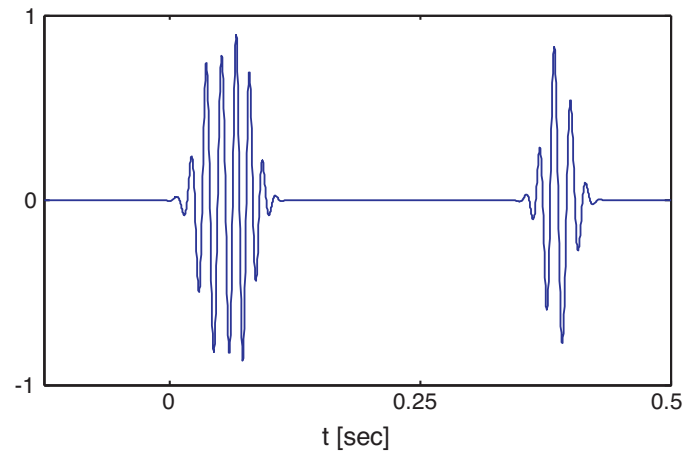

(c)

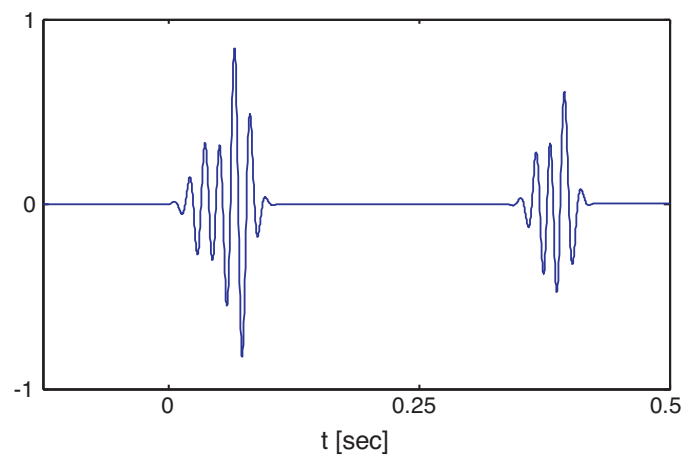

(b)

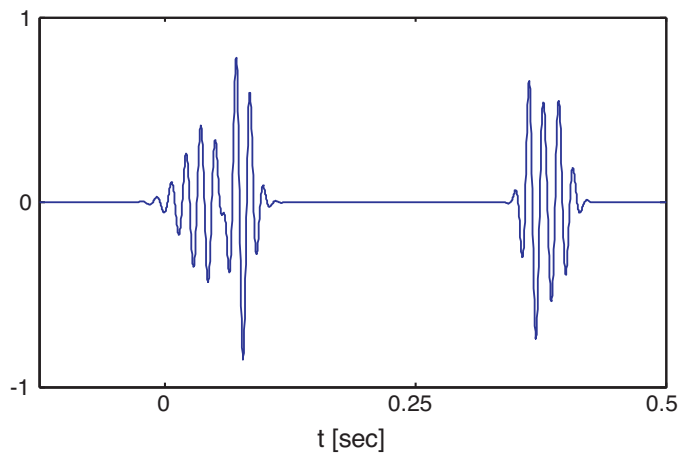

(d)

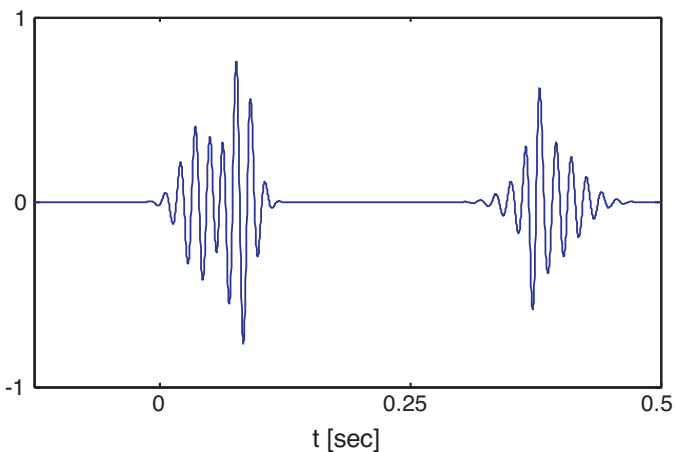

Fig. 7. Synthetic phonocardiogram (PCG) signals including two heart sounds, S1 and S2, generated by PCG dynamical model. Each heart sound waveform is generated by using two Gabor kernels whose parameters are listed by figure index (a), (b), (c) and (d) in Table 3 . The heartbeat duration is assumed to be $1 \mathrm{~s}$ and therefore the angular velocity $\omega$ would be equal to $2 \pi$. The origin of the time axis corresponds to the R-peak in the synchronous ECG signal.

\subsection{Denoising}

In order to evaluate the performance of the proposed denoising method, we should compare its results with those of conventional PCG denoising schemes, like WD, Ensemble

(a)

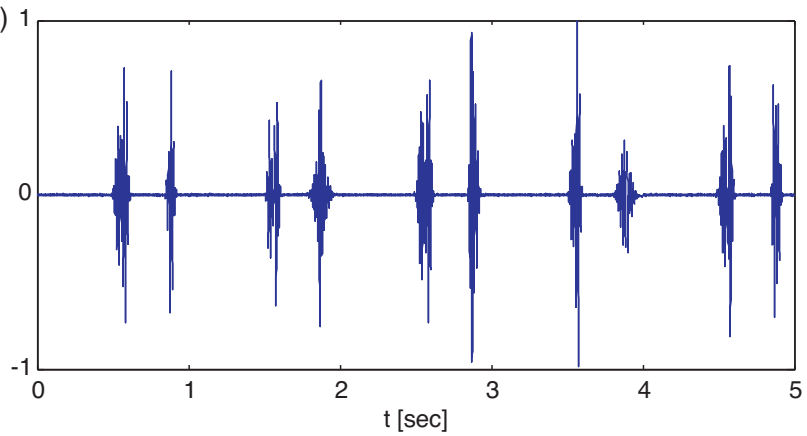

(b)

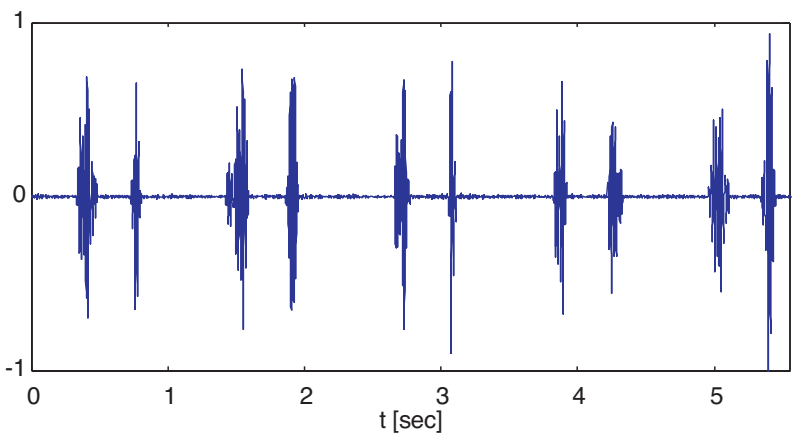

Averaging (EA), and Frequency Filtering. In the context of PCG denoising, WD has shown the best results [16], so we employ this method for comparison.

In conventional WD schemes, the type of the mother wavelet, shrinkage rule, hard- versus soft-thresholding, noise level rescaling approach, and number of decomposition levels must be selected. There are of course, some general rules concerning the selection of these parameters. For example, the mother wavelet is usually selected based on the resemblance to desired signal, or rescaling approach and shrinkage rules are selected according to the nature (white or colored) and variance of the noise. Messer et al. [16] have investigated the combinations of these parameters for PCG denoising. According to this study, among the different tested wavelets, Coiflet 4 and 5, Daubechies 11, 14, 20, and Symlet 9, 11,14, and among the different combinations, the Stein's Unbiased Risk Estimate (SURE) shrinkage rule and a soft-thresholding strategy and single level rescaling approach gave the superior results. The best decomposition level ranged from 5 to 10 , and more than 10 levels of decomposition showed no significant enhancement in the results. In this paper, the results of WD are based on the use of Coiflet5 wavelet with 10 levels of decomposition, which has shown the best performance among the other combinations in our dataset.

The quantitative comparison of the performance of different denoising methods is provided by comparing the amount of the signal-to-noise ratio (SNR) improvement achieved for the input signal which is defined as

Fig. 8. Comparison between (a) synthetic phonocardiogram (PCG) with additive white Gaussian noise, and (b) real PCG from a healthy person. 
(a)

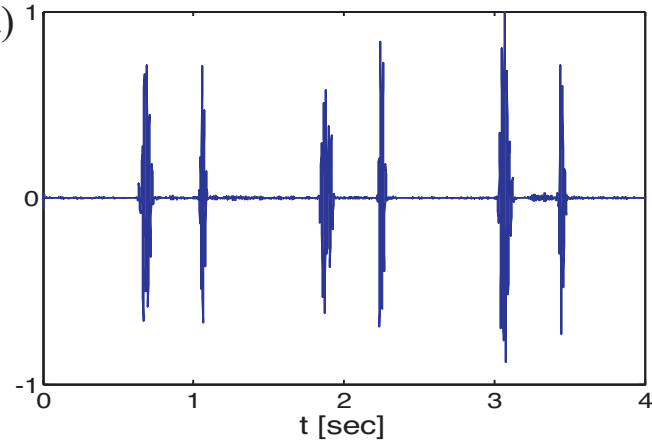

(c)

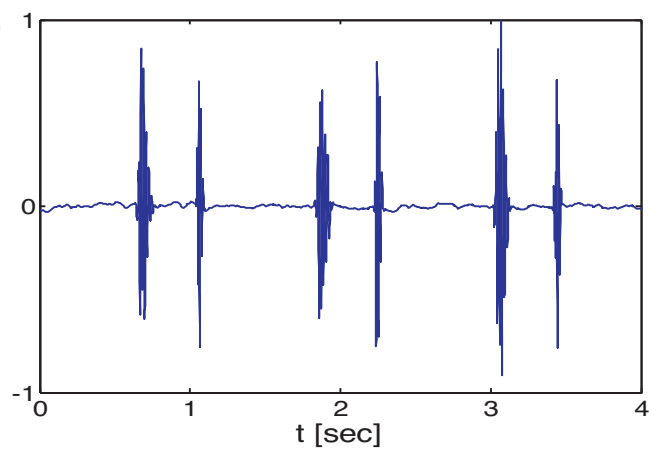

(b)

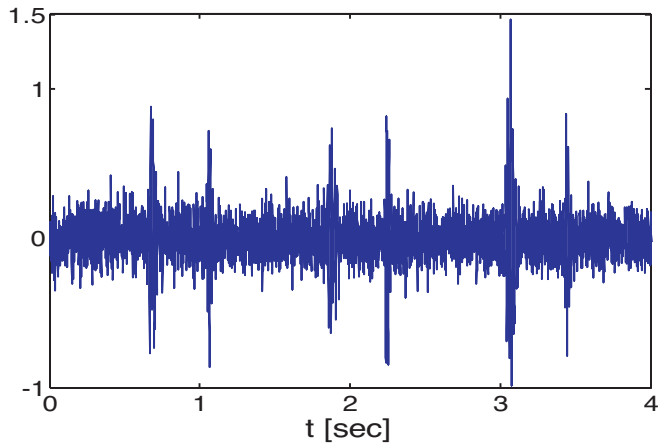

(d)

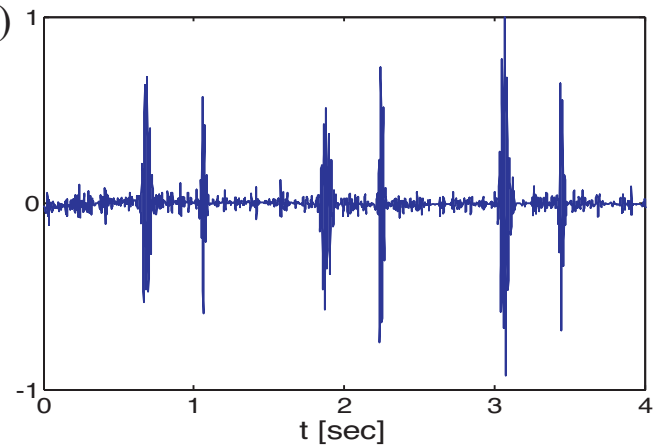

Fig. 9. Typical denoising results for an input signal of $0 \mathrm{~dB}$. (a) original, (b) noisy, (c) extended Kalman smoother (EKS), and (d) WD.

where SNRout and SNRin are the SNR of the output signal (in decibels) and the SNR of the input signal (in decibels), respectively, which are defined as follows:

$$
\begin{aligned}
& \text { SNRin }=10 \log \frac{\|x[n]\|}{\|N[n]\|}=10 \log \frac{\|x[n]\|}{\left\|x_{n}[n]-x[n]\right\|} \\
& \text { SNRout }=10 \log \frac{\|x[n]\|}{\|\hat{x}[n]-x[n]\|}
\end{aligned}
$$

where $x[n]$ is a test signal and $x_{n}[n]=x[n]+N[n]$ is the noisy signal obtained by adding a noise $N[n]$ with an appropriate variance to the test signal. The $x_{n}[n]$ is the input signal to the denoising algorithm, and $\hat{x}[n]$ is its output.

The synthetic white Gaussian noise with different variances were generated and added to the PCG segments, and the noisy signals with different SNRs were processed using the proposed method and the wavelet method. The whole procedure was repeated over the 30 PCG segments, and each time a different random noise was added to the signal. The reported SNR improvement was averaged over the whole 30 results and to ensure that the transient state of the filter has no influence on the results, the first $20 \%$ of the signal was not included in SNR calculation. In Fig. 9, typical results of the EKS and WD are illustrated for an input signal of $0 \mathrm{~dB}$. By visual inspection, it can be seen that EKS shows better performances and demonstrates the smoother result with respect to WD.

The main strength of the proposed denoising method is the knowledge it has about the PCG signal and its contents. This $a$ priori knowledge is achieved from the dynamic of the signal and provides an appropriate tool for extracting the basic components of the normal PCG form the all unwanted components that may exist in the signal. The traditional WD decomposes signal into different subspaces and then puts thresholds on them. So, some unwanted components (particularly those with high amplitude and wide frequency range) may pass through thresholding stage and appear in the denoised signal. In this case, WD is incapable of eliminating the unwanted components, since it has no sense about the certain events in the PCG (Fig. 9(d)). In contrast, EKS is quite capable of removing such components.

In addition to the environmental and recording noises, there may be some source of artifacts such as breath or lung sounds, which have quite different nature from white Gaussian noise. Although analyzing the nature of these sounds (breath and lung sounds) and how they affect heart sounds require another independent study (which is out of current study), however we claim that the proposed EKS structure is capable of removing such artifacts. In order to justify the capability of the proposed framework to remove such artifacts, it may suffice to illustrate the results qualitatively in Fig. 10. As it is seen, EKS structure has successfully removed the breath sounds contaminating heart sounds. The reason is that EKS is based upon the proposed model for fundamental components of PCG signal (S1 and S2 sounds) and therefore is able to extract these components (heart sounds) from other components such as breath sounds, which follow different underlying dynamics.

For a quantitative comparison, the mean and standard deviation (SD) of the SNR improvement versus input SNRs, achieved over the 30 PCG segments are plotted in Fig. 11. In low SNR scenarios, EKS demonstrates the best performance, and in high SNR scenarios, EKS and WD have somehow similar outputs. It is seen that WD has the least standard deviation over the different PCG segments.

The results are obtained using fixed covariance matrices $Q_{k}$ and $R_{k}$ for all PCG signals with the same input SNRs; although 

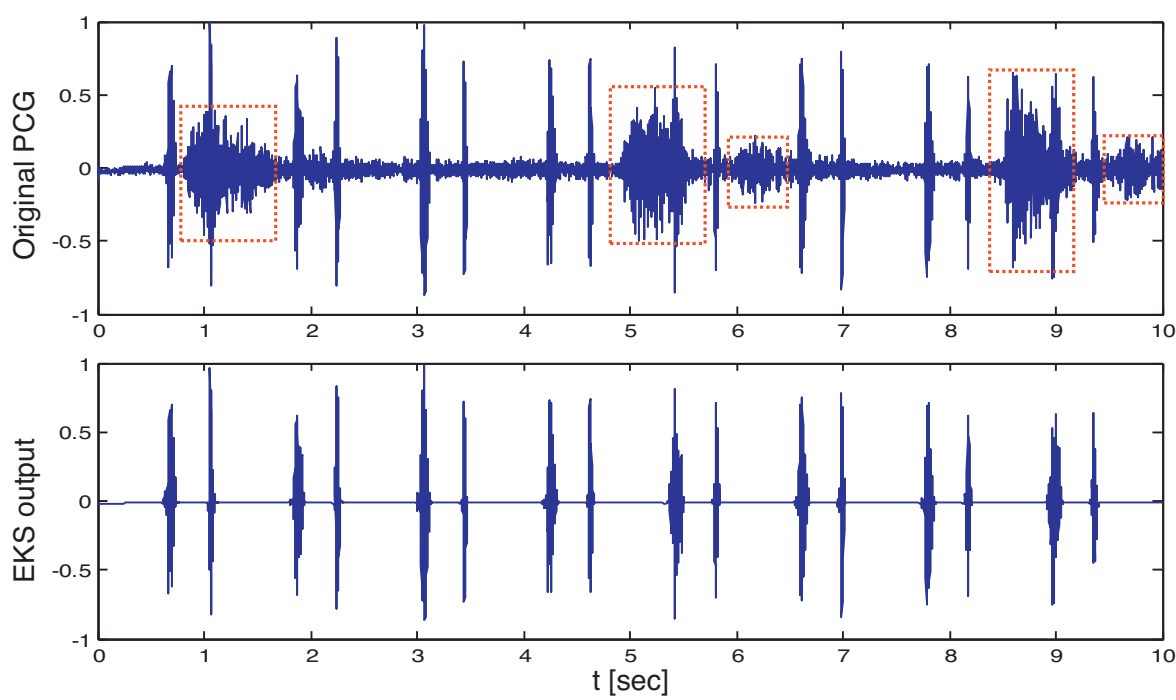

Fig. 10. The result of removing breath sounds from phonocardiogram (PCG) signal by the introduced extended Kalman smoother (EKS) structure; original PCG signal associated with breath sounds in dot-rectangular shapes (top), and output of the EKS structure (bottom). As it is clear, the breath sounds contaminating PCG signal are completely removed.

tuning the $Q_{k}$ and $R_{k}$ for each person, may enhance the results of the filter. But since WD has no signal-dependent tuning parameter, we used this policy to make the filter analogous with WD.

There is a trade-off between the number of Gabor kernels used for modeling each heart sound and the complexity of the denoising algorithm. The number of kernels used for each heart sound usually depends on the heart sounds waveform and are chosen from three to five kernels in this paper.
For analysis of the quality of the denoised PCG, two sets of six recordings with respectively a SNR equal to $15 \mathrm{db}$ and $20 \mathrm{db}$, are denoised and submitted to a senior cardiologist expert in the phonocardiography. After listening to both original and restored recordings, the expert has confirmed that the denoised PCGs are not suffering from any distortion and the restitution of the main components of the PCG is successful.
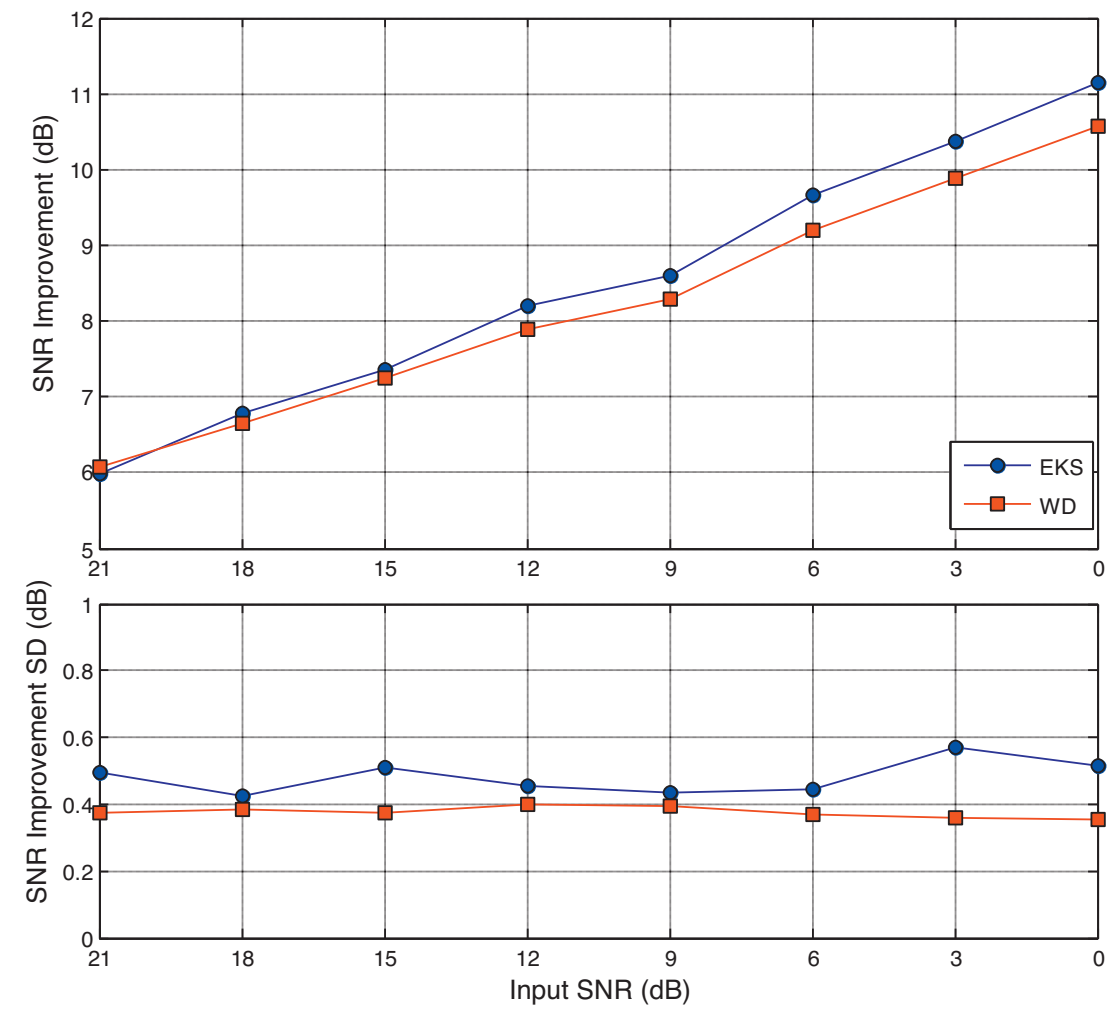

Fig. 11. The mean (top) and standard deviation (bottom) of the output SNR improvement versus input SNRs for our model-based Bayesian phonocardiogram (PCG) denoising and Wavelet denoising. 


\section{Conclusion and future works}

In this paper, we introduced a new dynamical model for PCG, which is capable of synthesizing realistic PCG signals. The introduced model is based on PCG morphology and model parameters may be chosen to generate various valid morphologies for synthesized heart sound waveforms. The sampling frequency and beat-to-beat intervals can also be adjusted. Having access to a realistic PCG provides a benchmark for testing numerous signal-processing techniques. A number of applications are perceivable for the model:

- by fitting the model to the morphology of various normal PCGs, and extracting their model parameters, a database of realistic PCGs could be created;

- by finding the pattern or relation among model parameters of one's normal heart sounds, abnormal morphologies could be detected;

- the synthetic PCG could be used to evaluate the effectiveness of different techniques for denoising, heart sound segmentation and compression;

- model-based PCG processing framework for different aims can be introduced.

Then, according to the latest application mentioned above, we established a new PCG denoising framework based on the proposed PCG dynamical model. The Bayesian denoising framework presented in this study was based on combining the Kalman filter structure with the introduced dynamical model. The results of the proposed denoising method were compared with those of WD. The results of the EKS demonstrate that the proposed model-based framework may serve as a novel approach to retrieve clean PCG from a noisy one. On the other hand, the EKS method has the superiority of real-time PCG processing and the fixed-lag smoother is usually more appropriate for this purpose.

Furthermore, since ECG model-based processing via Bayesian filters has been developed recently, this framework may also be very helpful to joint ECG-PCG real-time processing for clinical application.

\section{Acknowledgements}

The authors would like to thank Dr. Reza Sameni, Dr. Omid Sayadi and Dr. Jean-Marc Schleich for their valuable help and assistance.

\section{References}

[1] McSharry PE, Clifford GD, Tarassenko L, Smith LA. A dynamical model for generating synthetic electrocardiogram signals. IEEE Trans Biomed Eng 2003;50(3):289-94.

[2] Sameni R, Shamsollahi MB, Jutten C, Clifford GD. A nonlinear Bayesian filtering framework for ECG denoising. IEEE Trans Biomed Eng 2007;54(12):2172-85

[3] Sayadi O, Shamsollahi MB. ECG denoising and compression using a modified extended Kalman filter structure. IEEE Trans Biomed Eng 2008;55(9):2240-8.

[4] Sayadi O, Shamsollahi MB. A model-based Bayesian framework for ECG beat segmentation. Physiol Meas 2009;30(3): $335-52$.

[5] Almasi A, Shamsollahi MB, Senhadji L. A dynamical model for generating synthetic phonocardiogram signals. In: Proceedings of the 33rd International Conference of the IEEE Engineering in Medicine and Biology Society (EMBS). 2011 [5686-9].

[6] Grewal MS, Andrews AP. Kalman filtering theory and practice using MATLAB. 2nd ed. John Wiley \& Sons; 2001.

[7] Shen MF, Sun LS. Modelling and processing of phonocardiogram via parametric bispectral approach. In: Proceedings of the First Regional Conference IEEE Engineering in Medicine and Biology Society (EMBS) and 14th Conference of the Biomedical Engineering Society of India. 1995.

[8] Shen M, Shen F. Analysis of phonocardiographic signals based on higherorder spectra. In: Proceedings of The 3rd International Conference on Signal Processing. 1996.

[9] Chen D, Durand LG, Lee HC. Time-frequency analysis of the first heart sound. Part 1: Simulation and analysis. Med Biol Eng Comput 1997;35(4):306-10.

[10] Xu J, Durand LG, Pibarot P. Nonlinear transient chirp signal modeling of the aortic and pulmonary components of the second heart sound. IEEE Trans Biomed Eng 2000;47(10):1328-34.

[11] Xu J, Durand LG, Pibarot P. Extraction of the aortic and pulmonary components of the second heart sound using a nonlinear transient chirp signal model. IEEE Trans Biomed Eng 2001;48(3):22783.

[12] Sava H, Pibarot P, Durand LG. Application of the matching pursuit method for structural decomposition and averaging of phonocardiographic signals. Med Biol Eng Comput 1998;36(3):302-8.

[13] Wang W, Guo Z, Yang J, Zhang Y, Durand LG, Loew M. Analysis of the first heart sound using the matching pursuit method. Med Biol Eng Comput 2001;39(6):644-8.

[14] Wang W, Pan J, Lian H. Decomposition and analysis of the second heart sound based on the Matching Pursuit method. In: Proceedings of The 7th International Conference on Signal Processing. 2004.

[15] Zhang X, Durand LG, Senhadji L, Lee HC, Coatrieux JL. Analysissynthesis of the phonocardiogram based on the matching pursuit method. IEEE Trans Biomed Eng 1998;45(8):962-71.

[16] Messer SR, Agzarian J, Abbott D. Optimal wavelet denoising for phonocardiograms. Microelectr J 2001;32(12):931-41. 